\title{
Quaderni
}

QUADERNI Communication, technologies, pouvoir

67 | Automne 2008

Jeu vidéo et discours

\section{Dictionnaire des mondialisations, dirigé par Cynthia Ghorra-Gobin}

Anne-Laure Amilhat Szary

\section{(2) OpenEdition}

Édition électronique

URL : http://journals.openedition.org/quaderni/244

DOI : 10.4000/quaderni.244

ISSN : 2105-2956

Éditeur

Les éditions de la Maison des sciences de l'Homme

Édition imprimée

Date de publication : 5 octobre 2008

Pagination : 125-128

Référence électronique

Anne-Laure Amilhat Szary, "Dictionnaire des mondialisations, dirigé par Cynthia Ghorra-Gobin »,

Quaderni [En ligne], 67 | Automne 2008, mis en ligne le 13 décembre 2012, consulté le 23 septembre

2020. URL : http://journals.openedition.org/quaderni/244 ; DOI : https://doi.org/10.4000/quaderni.244

Tous droits réservés 


\section{Livre}

\section{dictionnaire des mondialisations}

\author{
dirigé par \\ Cynthia Ghorra-Gobin
}

Armand Collin, 2006
Il est à Paris une librairie spécialisée qui ne vend que des dictionnaires*. Preuve, s'il en était besoin, que l'on peut probablement faire un dictionnaire de toute chose suffisamment complexe pour être décomposée en une multitude de facettes méritant chacune explication. Le parti pris du collectif d'auteurs dirigés par C. Ghorra-Gobin dans l'ouvrage récemment publié par A. Colin, de définir la mondialisation comme un phénomène complexe et récent justifiait donc l'aventure éditoriale. L'effort produit par le collectif mobilisé ici est novateur et doit être considéré comme une étape dans la structuration des discours sur ce processus. Le dictionnaire réussit ainsi à éviter les écueils idéologiques, parvenant à ne faire primer ni la défense d'un néo-libéralisme triomphant ni les dénonciations alter-mondialistes. Notre compte rendu de lecture réunit cependant la formulation de critiques nombreuses vis-à-vis de l'ouvrage, conçues comme une exigence de précision toujours plus importante quant aux discours tenus sur la mondialisation.

Le terme est mis au pluriel dans le titre, pour insister le plus possible sur la diversité des situations induites par l'intensification et l'extension (spatiale) des échanges de biens, de services, d'idées, d'hommes etc. à l'échelle mondiale. Cette accélération des interrelations sociales, économiques, politiques et culturelles à la surface de la planète observée depuis la fin des années 1980, a conduit à un approfondissement des interdépendances, entre points du globe comme entre acteurs. Le pluriel accolé ici au terme de mondialisation correspond ainsi au souci de ne pas se référer à une période historique uniquement, mais à un ensemble de processus tant sociaux qu'économiques ou géographiques, 
souvent caractérisés de « globaux » ou « globalisants ». La mondialisation entre alors dans cette catégorie d'objets totaux ou totalisants dont la compréhension peut être facilitée par une analyse décomposée comme celle que permet un dictionnaire. Au risque de faire disparaître ou d'atténuer les interrelations entre les éléments du système, nous y reviendrons.

Pour contrebalancer l'ouverture thématique des articles, l'ouvrage repose sur une introduction solide, sorte de manifeste qui permet de positionner le collectif d'auteurs dans son champ de références bibliographiques, principalement occidentales (Europe et Amérique du Nord) sur le sujet. C'est un premier regret face à l'ouvrage qu'il ne donne pas un peu plus la voix aux penseurs des Suds (ne serait-ce qu'Amartya Sen) sur un processus qui les concerne et dont les écrits nous sont indispensables pour notre conceptualisation du global. Ceci-dit, le cheminement itératif de la démonstration confirme que le collectif d'auteurs mobilisés maîtrise les tenants et aboutissants du processus de mondialisation, perçu tant dans sa diversité planétaire que dans la multiplicité des approches disciplinaires, une autre façon de justifier le « $\mathrm{s}$ » final du titre. Pourtant, ce texte programmatique de qualité ne me semble pas relayer de façon suffisamment satisfaisante par le corps du dictionnaire, qui est loin d'en reprendre toutes les pistes.

Cette introduction pointe en effet un grand absent des discours sur la mondialisation, le travail, ce qui semble un point très pertinent, néanmoins cette remarque liminaire n'est pas suivie d'article « travail ».
On peut alors se demander quel est l'objectif des faiseurs de dictionnaires ? Il s'agit pour eux de « recueillir les mots d'une langue ou d'un art pour tenter d'en réunir les significations » (selon la définition proposée par ... un dictionnaire classique, le Petit Robert). Le lecteur se trouve alors en décalage avec le projet initial, tel que nous le percevons du moins. Pour moi, il ne s'agit pas d'un dictionnaire, mais plutôt d'un dictionnaire encyclopédique, voire d'une encyclopédie. Un dictionnaire constitue certes une boîte où l'on rangerait les mots pour faire voir ce qu'ils ont à l'intérieur d'eux (ce qu'ils pensent et reflètent), mais aussi un livre où le recueil des mots permet de mettre les choses qu'ils signifient en mouvement, dans une dynamique interne que l'on peut a posteriori qualifier d'ancêtre de l'hypertexte. L'encyclopédie a la même prétention à l'exhaustivité, mais d'un point de vue de la théorie plus que du sens : il s'agit d'y réunir des connaissances pour qu'elles s'éclairent les unes les autres, comme le soleil qui éclaire une à une les choses découvertes par les hommes, accompagnant l'enfant faisant le tour des notions au fil de son éducation. C'est pourquoi, si l'on ne peut que se satisfaire de la volonté de mettre en œuvre un dictionnaire des mondialisations, terme équivalent - d'une certaine façon - à celui de complexité, on attend cependant d'un ouvrage de ce type qu'il puisse non seulement éclairer le sens mais aussi mettre en lumière les interrelations, rendre visibles les liens et relations de causalité ou du moins les interférences constantes entre les termes de la mondialisation. Là encore, il me semble que le résultat soit partiel: chaque entrée est un bloc, qui ne fonctionne pas assez en résonance avec les autres. Certes, à la fin de chaque rubrique, on trouve des renvois, mais ce Dictionnaire des mon- 
dialisations semble construit sur une architecture somme toute trop traditionnelle pour un cerner objet social de nature différente, et on imaginerait volontiers la prolongation de ce travail sur un support électronique. La structure des rubriques est originale, elle fait alterner paragraphes de définition et de débat autour de chaque mot analysé, les entrées étant pour certaines accompagnées d'essais au statut incertain. Malgré leur apparente neutralité, les premières parties de chacune des entrées posent certains soucis, s'attardant un peu longuement sur les définitions littérales (les bases informatiques du « Virtuel », le sens donné par Mc Luhan au « Global village » au détriment des usages contemporains du mot), et pas assez sur la problématique induite par l'introduction du terme traité par le dictionnaire dans les réseaux de la mondialisation. De ce point de vue, certaines notices sont plus réussies (« Régulation », « subsidiarité ») que d'autres ( Centre / Périphérie », longue notice qui déqualifie ce vieux modèle sans véritablement justifier son abandon, citant $P$. Veltz mais pas le concept essentiel de découplage du centre et de la périphérie que ce dernier a défini dans Ville et mondialisation-1996). On trouve des surprises: les entrées «communisme» (marxisme n'aurait-il pas été plus pertinent?) et «sous-développement» mais pas l'item « Suds », un des grands absents.

L'ouvrage pose enfin le problème de son lectorat. Son insertion dans une collection des éditions Colin indique une préférence pour un public universitaire or on aurait pu souhaiter, sur un thème aussi englobant, un mode d'écriture destiné à une plus vaste audience. Tel qu'il est conçu cependant, il n'est destiné ni aux tout à fait néophytes ni aux spécialistes.
Cette pluridisciplinarité reste cependant l'une des plus belles réussites du dictionnaire, qui permet notamment de faire reconnaître, aux côtés de la géographie dominante, une approche renouvelée des relations internationales (cf. notamment les entrées «Reconnaissance mutuelle », « justice internationale »). On trouvera toujours que cette ouverture aurait pu être menée plus loin : il nous semble que certains termes de la mondialisation économique et financière brillent par leur absence (on aurait souhaité un traitement plus approfondi d'une entrée comme celle de «bourse», et la présence d'entrées comme « fusion-acquisition », «OPA », « junk bonds », « fonds de pension », etc. ). Et surtout ces mondialisations restent très désincarnées, à une distance trop lointaine d'une prise en compte sociale des mondialisations (certes, figurent « Société Civile, puis «Économie informelle », «Fragmentation spatiale », mais pas «spatiale »; le risque est présenté de façon thématique, à travers ses volets économique, environnemental et systémique, mais pas la société du risque n'apparaît qu'au travers du « principe de précaution»). Des types d'entrées auraient pu être établis (lieux, processus et acteurs) qui auraient pu faciliter la mise en résonance des entrées entre elles. Parmi les acteurs on lit avec intérêt une notice comme celle de "Société militaire privée (SMP) », mais rien sur l'OMC ou d'autres acteurs globaux émergents (le Brésil et le G22) ou encore des organisations comme ATTAC ou ses équivalents nord ou sud -américains. Le contexte intellectuel de mise en place des mondialisations aurait également gagné à être creusé, et pourtant pas d'article sur la postmodernité si sur le « cultural turn » / « tournant culturel ».

Du point de vue de la lecture de l'espace, qu'en 
est-il d'un Dictionnaire dirigé par une géographe ? Les entrées font une belle part au multiscalaire, mais pas forcement à toute la complexité des interactions induites. L'entrée « Agriculture » par exemple ne pousse pas de façon assez satisfaisante le lien production / consommation, amenant à passer sous silence les filières biologiques, questionnant le bouleversement des filières. Dans le monde urbain, la définition de la ville ne laisse de surprendre : livrée comme un "morceau » de territoire, elle apparaît de façon généalogique, dans l'opposition des modèles européen / et anglo-saxon (selon une différenciation des fonctions, plus politiques en Europe, plus économiques dans monde nord-américain et anglais) ; le reste de la notice reste dans le flou, les notions de ville « globale », « méga », « émergente », « diffuse », « éclatée », « fragmentée » (ici dans une réduction à la multiplication de municipalités qui composent une agglomération), «villegateway », « ville État » figurant sous la forme d'appellations cataloguées mais non explicitées, accumulation de termes manquant de problématisation. Ou parfois de précision, notamment chiffrée : dire, à propos du « village global », que « la fracture numérique est sérieuse » suffit-il vraiment? À une autre échelle, une entrée comme celle de « régionalisation » manque là aussi de la précision sur les modalités du processus (possible notamment grâce à la littérature anglo-saxonne sur le régionalisme ouvert).

Aborder les mondialisations implique d'abandonner toute prétention à l'exhaustivité, menant à des choix qui seront sans doute cause de regret pour certains lecteurs, paradoxe et risque que les auteurs assument dès les premières pages. Depuis le texte introductif jusqu'aux rubriques traitées, le
Dictionnaire des Mondialisations constitue ainsi une somme de connaissances pluridisciplinaires dont les étudiants sauront tirer profit dans leur travail personnel dans le cadre d'approches très distinctes, et à ce titre l'ouvrage trouve bien sa place dans une collection très ambitieuse pour les sciences sociales.
* La Maison des Dictionnaires, bd du Montparnasse, propose un catalogue de plus de 4000 références. 